\title{
STRONG COMPACTNESS AND STATIONARY SETS
}

\author{
JOHN KRUEGER
}

\begin{abstract}
We construct a model in which there is a strongly compact cardinal $\kappa$ such that the set $S\left(\kappa, \kappa^{+}\right)=\left\{a \in P_{\kappa} \kappa^{+}\right.$: o.t. $\left.(a)=(a \cap \kappa)^{+}\right\}$is non-stationary.
\end{abstract}

If $\kappa$ is a $\kappa^{+}$-supercompact cardinal then the set $S\left(\kappa, \kappa^{+}\right)=\left\{a \in P_{\kappa} \kappa^{+}\right.$: o.t. $\left.(a)=(a \cap \kappa)^{+}\right\}$is stationary. A natural question to ask is whether the $\kappa^{+}$strong compactness of a cardinal $\kappa$ implies the stationarity of $S\left(\kappa, \kappa^{+}\right)$. In the present paper we solve this problem by constructing a model in which there is a strongly compact cardinal $\kappa$ such that $S\left(\kappa, \kappa^{+}\right)$is non-stationary.

We use two main techniques in our consistency proof. First, we use our forcing poset from [6] for destroying stationary subsets of $P_{\kappa} \kappa^{+}$using partial square sequences. Secondly, we use a Magidor iteration of Prikry forcing.

The method of argument we present has applications to other problems. For example, in the final section we construct a model in which there is a strongly compact cardinal $\kappa$ with $2^{\left(\kappa^{+}\right)}=\kappa^{+++}$, but for a club of $\alpha<\kappa, 2^{\left(\alpha^{+}\right)}=\alpha^{++}$.

The contents of the paper are as follows. In Section 1 we outline preliminaries and notation. Section 2 describes our method of using partial square sequences to destroy stationary sets. Section 3 provides some additional background material on large cardinals and forcing. Section 4 gives a summary of the consistency proof. In Section 5 we complete the first step of the consistency proof by iterating the partial square poset. In Section 6 we finish the proof using a Magidor iteration of Prikry forcing. In Section 7 we give another application of our method to prove a consistency result concerning strong compactness and $\mathrm{GCH}$.

I would like to thank Moti Gitik for discussing this material with me.

§1. Preliminaries. We assume that the reader is familiar with iterated forcing, Prikry forcing, and supercompact cardinals; see [1], [2], and [5].

If $\kappa$ is regular and $\kappa \subseteq X$, define $P_{\kappa} X$ as the set of $a \subseteq X$ such that $|a|<\kappa$ and $a \cap \kappa \in \kappa$. A set $C \subseteq P_{\kappa} X$ is club if it is closed under unions of $\subseteq$-increasing sequences with length less than $\kappa$, and for all $a$ in $P_{\kappa} X$ there is $b \in C$ with $a \subseteq b$. A set $S \subseteq P_{\kappa} X$ is stationary if for every club $C, S \cap C$ is non-empty.

The expression " $\theta \gg \kappa$ " indicates that $\theta>2^{2^{|H(\kappa)|}}$. If $\theta \gg \kappa$ and $\mathcal{A}$ is a structure with underlying set $H(\theta)$, then the collection of elementary substructures of $\mathcal{A}$ in $P_{\kappa} H(\theta)$ is a club set.

This work was supported by FWF project number P16790-N04. 
An ultrafilter $U$ on $P_{\kappa} X$ is normal if for every function $f: P_{\kappa} X \rightarrow X$ which is regressive (i.e. $f(a) \in a$ for all $a$ ) there is a set $A$ in $U$ such that $f \uparrow A$ is constant. We assume our ultrafilters are non-principal and fine; that is, $\{a\} \notin U$ for all $a$, and for any $i$ in $X$, the set $\left\{a \in P_{\kappa} X: i \in a\right\}$ is in $U$.

Suppose that $\kappa \leq \lambda$ are cardinals. Then $\kappa$ is $\lambda$-supercompact if there exists a normal ultrafilter on $P_{\kappa} \lambda$, or equivalently, there exists an elementary embedding $j: V \rightarrow M$, where $M$ is a transitive inner model of ZFC, such that $\operatorname{crit}(j)=\kappa$, $j(\kappa)>\lambda$, and ${ }^{\lambda} M \subseteq M$. We say that $\kappa$ is $\lambda$-strongly compact if there exists a $\kappa$-complete ultrafilter on $P_{\kappa} \lambda$, or equivalently, there is an elementary embedding $j: V \rightarrow M$, where $M$ is a transitive inner model of ZFC, such that $\operatorname{crit}(j)=\kappa$, $j(\kappa)>\lambda$, and there exists a set $a$ in $M$ with $|a|<j(\kappa)$ such that $j$ " $\lambda \subseteq a$. A cardinal $\kappa$ is supercompact (respectively, strongly compact) if for all $\lambda>\kappa, \kappa$ is $\lambda$-supercompact (respectively, $\lambda$-strongly compact).

If $a$ is a set of ordinals then o.t.( $a$ ) denotes the order type of $a$. If o.t.( $a$ ) is a limit ordinal, $\operatorname{cf}(a)$ denotes $\operatorname{cf}($ o.t. $(a))$. Note that $\operatorname{cf}(a)=\operatorname{cf}(\sup a)$.

If $\theta \gg \kappa$ and $N \prec H(\theta)$ is in $P_{\kappa} H(\theta)$, then $N \cap \kappa^{+}$contains as a member any limit point below $\sup \left(N \cap \kappa^{+}\right)$which has cofinality different from $\operatorname{cf}(N \cap \kappa)$.

If $M \subseteq N$ are transitive models of set theory with the same ordinals and $\lambda$ is a cardinal, we say that $M$ is $\lambda$-closed in $N$ if ${ }^{\lambda} M \cap N \subseteq M$. The model $M$ is $\lambda$-closed in $N$ iff ${ }^{\lambda} O n \cap N \subseteq M$.

We will use the phrase forcing poset to indicate any ordering $\langle\mathbb{P}, \leq\rangle$ whose relation $\leq$ is reflexive and transitive. For a detailed discussion of the author's conventions concerning forcing posets, separative posets, and Boolean algebras, see [7].

If $\mathbb{P}$ is a forcing poset and $p$ is a condition in $\mathbb{P}$, we write $\mathbb{P} / p$ for the forcing poset with underlying set $\{q \in \mathbb{P}: q \leq p\}$, ordered the same as in $\mathbb{P}$.

Suppose $\varphi$ is a statement in the forcing language for a poset $\mathbb{P}$. We write ${ }^{0} \varphi$ for $\varphi$ and ${ }^{1} \varphi$ for $\neg \varphi$.

If $\lambda$ is an ordinal, a canonical $\mathbb{P}$-name for a subset of $\lambda$ is a name of the form $\left\{\langle p, \check{\alpha}\rangle: p \in A_{\alpha}, \alpha<\lambda\right\}$, where each $A_{\alpha}$ is an antichain. If $p$ forces that $\dot{X}$ is a subset of $\lambda$, then there is a canonical name $\dot{Y}$ for a subset of $\lambda$ such that $p$ forces that $\dot{X}=\dot{Y}$.

Suppose that $G$ is generic for a forcing poset $\mathbb{P}$ and $\varphi$ is a statement in the forcing language. If each $p$ in $G$ has an extension $q \leq p$ which forces $\varphi$, then $\varphi$ holds in $V[G]$.

A forcing poset $\mathbb{P}$ is $\lambda$-strategically closed for an ordinal $\lambda$ if there is a strategy for Player II in the following game: Player I starts the game by playing a condition $p_{1}$ in $\mathbb{P}$. Player II responds with a condition $p_{2} \leq p_{1}$. The game continues in this manner, each player choosing a condition below the previous one, with Player I playing at odd stages and Player II at even successor stages. At limit stages Player II plays a condition below all the conditions played so far. Player II wins if it is able to play a condition at all stages below $\lambda$.

For a regular cardinal $\alpha$ and an ordinal $\beta$, the forcing poset $\operatorname{Add}(\alpha, \beta)$ consists of conditions $p$ which are partial functions $\alpha \times \beta \rightarrow 2$ with size less than $\alpha$, ordered by $q \leq p$ if $p \subseteq q$. The poset $\operatorname{Add}(\alpha, \beta)$ is $\alpha$-closed and $\left(\alpha^{<\alpha}\right)^{+}$-c.c. If $G$ is generic for $\operatorname{Add}(\alpha, \beta)$ then $G$ codes a sequence of $\beta$ many new subsets of $\alpha$. 
A triple $\left\langle\mathbb{Q}, \leq, \leq^{*}\right\rangle$ is called a Prikry type forcing poset if $\langle\mathbb{Q}, \leq\rangle$ and $\left\langle\mathbb{Q}, \leq^{*}\right\rangle$ are forcing posets, $q \leq^{*} p$ implies $q \leq p$, and $\mathbb{Q}$ satisfies the Prikry property: for any $\varphi$ in the forcing language for $\langle\mathbb{Q}, \leq\rangle$ and for any $p$ in $\mathbb{Q}$, there exists $q \leq^{*} p$ such that $q$ decides $\varphi$. If $\alpha$ is a cardinal we say that $\mathbb{Q}$ is $\alpha$-weakly closed if $\left\langle\mathbb{Q}, \leq^{*}\right\rangle$ is $\alpha$-closed. We say that $\mathbb{Q}$ satisfies the direct extension property if whenever $q, r \leq^{*} p$, there is $s \leq^{*} q, r$. When we say that we force with a Prikry type forcing poset $\mathbb{Q}$, we always mean that we force with $\langle\mathbb{Q}, \leq\rangle$.

$\S 2$. Stationary sets and partial squares. Suppose that $\kappa$ is a weakly inaccessible cardinal. Then there is a club set of $a$ in $P_{\kappa} \kappa^{+}$such that o.t. $(a) \leq(a \cap \kappa)^{+}$ (see [7]). Define $S\left(\kappa, \kappa^{+}\right)$as the set of $a$ in $P_{\kappa} \kappa^{+}$such that o.t. $(a)=(a \cap \kappa)^{+}$.

In [6] we introduced the idea of a partial square sequence and showed how to use such a sequence to destroy certain stationary subsets of $S\left(\kappa, \kappa^{+}\right)$. This method will play a crucial role in the proof of the main theorem.

Let $B \subseteq \kappa^{+}$be a set of limit ordinals. We say that $\square_{\kappa}^{B}$ holds if there exists a partial square sequence $\left\langle c_{\alpha}: \alpha \in B\right\rangle$ satisfying:

(1) $c_{\alpha}$ is club in $\alpha$,

(2) if $\operatorname{cf}(\alpha)<\kappa$ then o.t. $\left(c_{\alpha}\right)<\kappa$,

(3) if $\beta$ is in $\lim \left(c_{\alpha}\right) \cap B$ then $c_{\beta}=c_{\alpha} \cap \beta$.

It is easy to force a partial square sequence using a generalization of Jensen's poset for adding a square sequence. Suppose that $B \subseteq \kappa^{+}$is an unbounded set of limit ordinals. Define $\mathbb{P}_{B}$ as follows. A condition in $\mathbb{P}_{B}$ is a sequence $\left\langle c_{\alpha}: \alpha \in B \cap(\beta+1)\right\rangle$, for some $\beta<\kappa^{+}$, satisfying (1), (2), and (3) above. We let $q \leq p$ if $p$ is an initial segment of $q$.

Note that $\mathbb{P}_{B}$ has size no larger than $2^{\kappa}$. The proof of the next lemma is basically the same as the proof of the corresponding fact for Jensen's poset for adding a square.

Lemma 2.1. The poset $\mathbb{P}_{B}$ is $(\kappa+1)$-strategically closed.

Proof. We describe a strategy by considering a run of the game. Suppose $\left\langle p_{i}: i<\beta\right\rangle$ is a run of the game up to stage $\beta$ and it is Player II's turn. For each $i<\beta$ let $\gamma_{i}$ be the least ordinal $\gamma$ so that $p_{i}$ is of the form $\left\langle c_{\alpha}: \alpha \in B \cap(\gamma+1)\right\rangle$. First assume $\beta=\alpha+1$. Let $\xi$ be the least element of $B$ larger than $\gamma_{\alpha}$, and define $p_{\beta}=p_{\alpha} \cup\left\{\left\langle\xi, c_{\xi}\right\rangle\right\}$, where $c_{\xi}$ is some club subset of $\xi$ with order type $\operatorname{cf}(\xi)$ and $\min \left(c_{\xi}\right)>\gamma_{\alpha}$.

Suppose that $\beta$ is a limit ordinal. Let $\gamma_{\beta}=\bigcup_{i<\beta} \gamma_{i}$. If $\gamma_{\beta}$ is not in $B$, then let $p_{\beta}=\bigcup\left\{p_{i}: i<\beta\right\}$. If $\gamma_{\beta}$ is in $B$, then define $p_{\beta}$ as $\bigcup\left\{p_{i}: i<\beta\right\} \cup\left\{\left\langle\gamma_{\beta}, c_{\gamma_{\beta}}\right\rangle\right\}$, where $c_{\gamma_{\beta}}=\left\{\gamma_{i}: i<\beta\right\}$. If $\beta<\kappa$ then $c_{\gamma_{\beta}}$ has order type less than $\kappa$. Suppose that $\gamma$ is a limit point of $c_{\gamma_{\beta}}$ in $B$. Then $\gamma=\gamma_{i}$ for some limit ordinal $i$ and $c_{\gamma}=\left\{\gamma_{j}: j<i\right\}=c_{\gamma_{\beta}} \cap \gamma$.

A standard argument shows the lemma implies that $\mathbb{P}_{B}$ does not add any subsets to $\kappa$. The lemma also implies that $\mathbb{P}_{B}$ adds a partial square sequence with domain $B$.

Now let us show how to use this forcing poset to destroy stationary subsets of $P_{\kappa} \kappa^{+}$. Let $B \subseteq \kappa^{+}$be an unbounded set of limit ordinals and $\mu_{0}, \mu_{1}$ distinct regular cardinals less than $\kappa$ such that $B$ contains all its limit points with cofinality either $\mu_{0}$ or $\mu_{1}$. 
Lemma 2.2. The poset $\mathbb{P}_{B}$ destroys the stationarity of the set $S=\{a \in$ $\left.S\left(\kappa, \kappa^{+}\right): \sup (a) \in B\right\}$.

Proof. Let $G$ be generic for $\mathbb{P}_{B}$ and let $\left\langle c_{\beta}: \beta \in B\right\rangle$ be the generic partial square sequence given by $G$.

Suppose for a contradiction that $S$ is stationary in $V[G]$. Fix $\theta \gg \kappa^{+}$regular. Then there is $N$ in $P_{\kappa} H(\theta)$ such that $N \cap \kappa^{+} \in S, N \cap \kappa$ is a cardinal, $\mu_{0}$ and $\mu_{1}$ are in $N$, and $N$ is an elementary substructure of

$$
\left\langle H(\theta), \in,\left\langle c_{\beta}: \beta \in B\right\rangle\right\rangle .
$$

Let $a=N \cap \kappa^{+}, \beta=\sup (a)$, and $\kappa_{a}=a \cap \kappa$. Since $a$ is in $S$, the ordinal $\beta$ is in $B$. Note that $\operatorname{cf}(\beta)=\kappa_{a}^{+}$. In particular, $\operatorname{cf}(\beta)>\mu_{0}, \mu_{1}$.

Let $\mu$ be one of $\mu_{0}$ or $\mu_{1}$ which is different from $\operatorname{cf}\left(\kappa_{a}\right)$. Then $a$ is closed under suprema of bounded subsets with order type $\mu$. By the choice of $B$ and the $(\kappa+1)$-strategic closure of $\mathbb{P}_{B}$, the set $B$ is also closed under suprema of subsets with order type $\mu$. By elementarity, $\beta$ is a limit point of $B$. So $a \cap B$ is a stationary subset of $\beta$.

Since $\operatorname{cf}(\beta)=\kappa_{a}^{+}$, o.t. $\left(c_{\beta}\right) \geq \kappa_{a}^{+}$. So there exists $\gamma$ in $a \cap B \cap \lim \left(c_{\beta}\right)$ such that o.t. $\left(c_{\beta} \cap \gamma\right) \geq \kappa_{a}$. Then $c_{\gamma}=c_{\beta} \cap \gamma$ has order type at least $\kappa_{a}$. But by elementarity, o.t. $\left(c_{\gamma}\right)$ is in $N \cap \kappa=\kappa_{a}$, which is a contradiction.

Note that since $\mathbb{P}_{B}$ is $(\kappa+1)$-distributive, the set $S$ in Lemma 2.2 satisfies the same definition in $V[G]$ as it does in $V$.

The poset $\mathbb{P}_{B}$ is simpler and easier to work with than previous posets for destroying stationary subsets of $P_{\kappa} \kappa^{+}$, as found in [4] and [7]. For more applications of the partial square poset see $[6]$.

§3. Background on large cardinals and forcing. In this section we give a summary of the background material on large cardinals and forcing which we use in our consistency proof. None of the results in this section are due to the author.

The following lemma is the main tool for extending elementary embeddings.

Lemma 3.1. Suppose that $j: M \rightarrow N$ is an elementary embedding between transitive models of set theory, $\mathbb{P}$ is a forcing poset in $M, G$ is generic for $\mathbb{P}$ over $M$, and $H$ is generic for $j(\mathbb{P})$ over $N$.

Then $j$ can be lifted to $j: M[G] \rightarrow N[H]$ such that $j(G)=H$ iff $j$ " $G \subseteq H$. In particular, $j$ can be lifted if there exists a condition $s$ in $H$ such that $s \leq j(p)$ for all $p$ in $G$.

Proof. If $j^{\text {" }} G \subseteq H$ then the map $j\left(\dot{x}^{G}\right)=j(\dot{x})^{H}$ is well-defined and satisfies the required properties.

We will use Silver's notation and refer to a condition $s$ in $j(\mathbb{P})$ such that $s \leq j(p)$ for all $p$ in $G$ as a master condition.

LEMma 3.2. Suppose that $U$ is a normal ultrafilter on a cardinal $\kappa$ and $j$ : $V \rightarrow M=\operatorname{Ult}(V, U)$. Let $\mathbb{P}$ be a forcing poset and let $G$ be a generic filter for $\mathbb{P}$ over $V$. Suppose that in $V[G]$ there is a generic filter $H$ for $j(\mathbb{P})$ over $M$ such that $j$ " $G \subseteq H$. Lift $j$ to $j: V[G] \rightarrow M[H]$. 
In $V[G]$ define $U^{*}$ by letting $X \in U^{*}$ iff $X \subseteq \kappa$ and $\kappa \in j(X)$. Then $U^{*}$ is a normal ultrafilter extending $U$ and $M[H]=\operatorname{Ult}\left(V[G], U^{*}\right)$.

Proof. We omit the standard argument that $U^{*}$ is a normal ultrafilter extending $U$. Since the only isomorphism between two transitive models of set theory is the identity mapping, it suffices to prove that $M[H]$ and $\operatorname{Ult}\left(V[G], U^{*}\right)$ are isomorphic.

Define $k: M[H] \rightarrow \operatorname{Ult}\left(V[G], U^{*}\right)$ as follows. Let $a$ be in $M[H]$. Then there is a $j(\mathbb{P})$-name $\dot{x}$ in $M$ such that $\dot{x}^{H}=a$. Since $\dot{x}$ is in $M$, we can choose $f: \kappa \rightarrow V$ such that $[f]=\dot{x}$. Moreover, choose $f$ so that $f(\alpha)$ is a $\mathbb{P}$-name for all $\alpha$. In $V[G]$ define $g: \kappa \rightarrow V[G]$ by letting $g(\alpha)=f(\alpha)^{G}$. Now let $k(a)=[g]$.

First we show that $k$ is well-defined. Suppose that $a=\dot{x}^{H}=\dot{y}^{H}$, where $\left[f_{\dot{x}}\right]=\dot{x}$ and $\left[f_{\dot{y}}\right]=\dot{y}$. Then there exists a condition $q$ in $H=j(G)$ which forces over $M$ that $\dot{x}=\dot{y}$. In $M$ let $q=[h]$ and write $h(\alpha)=p_{\alpha}$. Then there exists a set $A$ in $U$ such that for all $\alpha$ in $A, p_{\alpha}$ forces that $f_{\dot{x}}(\alpha)=f_{\dot{y}}(\alpha)$. In $M[H]$, $q$ is in $j(G)$. But $j(h)(\kappa)=q$ in $M$ and hence in $M[H]$. By definition of $U^{*}$, there is $A^{*} \subseteq A$ in $U^{*}$ such that for all $\alpha$ in $A^{*}, h(\alpha)=p_{\alpha}$ is in $G$. Then for all $\alpha$ in $A^{*}, f_{\dot{x}}(\alpha)^{G}=f_{\dot{y}}(\alpha)^{G}$. Define $g_{\dot{x}}$ and $g_{\dot{y}}$ by $g_{\dot{x}}(\alpha)=f_{\dot{x}}(\alpha)^{G}$ and $g_{\dot{y}}(\alpha)=f_{\dot{y}}(\alpha)^{G}$. Then for all $\alpha$ in $A^{*}, g_{\dot{x}}(\alpha)=g_{\dot{y}}(\alpha)$, so $\left[g_{\dot{x}}\right]=\left[g_{\dot{y}}\right]$. This proves that $k$ is well-defined.

A similar argument shows that $k$ is injective and that $a \in b$ iff $k(a) \in k(b)$. To show that $k$ is surjective, fix $[h]$ in $\operatorname{Ult}\left(V[G], U^{*}\right)$. Let $\dot{h}$ be a $\mathbb{P}$-name for $h$, and for each $\alpha$ let $\dot{b}_{\alpha}$ be a $\mathbb{P}$-name for $\dot{h}(\alpha)$. Then $\left[\alpha \mapsto \dot{b}_{\alpha}\right]$ is a $j(\mathbb{P})$-name in $M$. Let $a=\left[\alpha \mapsto \dot{b}_{\alpha}\right]^{H}$, which is in $M[H]$. Using the definition of $k$, it is straightforward to check that $k(a)=[h]$.

A standard way to extend an elementary embedding is to apply strategic closure to build a generic filter. Suppose that $M \subseteq N$ are transitive models of set theory and $\lambda$ is an $N$-cardinal. Let $\mathbb{P}$ be a forcing poset in $M$ and let $p$ be in $\mathbb{P}$. Suppose that $N$ models that $\mathbb{P}$ is $\lambda$-strategically closed and has no more than $\lambda$ many maximal antichains in $M$. Enumerate all maximal antichains in $M$ as $\left\langle A_{i}: i<\lambda\right\rangle$. Applying strategic closure we can inductively define a decreasing sequence $\left\langle p_{i}: i<\lambda\right\rangle$ so that $p_{0}=p$ and $p_{i+1}$ is below some member of $A_{i}$. This sequence of conditions generates a generic filter $H$ for $\mathbb{P}$ over $M$ which contains $p$.

The following two lemmas show how to verify closure of generic extensions.

LEMma 3.3. Suppose that $M \subseteq N$ are transitive models of set theory with the same ordinals and $\lambda$ is a regular uncountable cardinal in $N$ such that $M$ is $<\lambda$ closed in $N$. If $\mathbb{P}$ is a forcing poset in $M$ which is $\lambda$-c.c. in $N$ and $G$ is generic for $\mathbb{P}$ over $N$, then $M[G]$ is $<\lambda$-closed in $N[G]$.

Proof. We prove that ${ }^{<\lambda} O n \cap N[G] \subseteq M[G]$. Suppose that $p$ is in $G$ and $p$ forces over $N$ that $\dot{f}: \beta \rightarrow O n$ for some $\beta<\lambda$. For each $\alpha<\beta$ let $A_{\alpha}$ be a maximal antichain below $p$ contained in the dense set of conditions which decide the value of $\dot{f}(\alpha)$. Let $X_{\alpha}$ be the set of pairs $\langle q, \gamma\rangle$ such that $q \in A_{\alpha}$ and $q$ forces over $N$ that $\dot{f}(\alpha)=\gamma$. Then $\left|A_{\alpha}\right|=\left|X_{\alpha}\right|<\lambda$, and so $\left\langle A_{\alpha}, X_{\alpha}: \alpha<\beta\right\rangle$ is in $M$. Define a name $\dot{g}$ in $M$ by letting $\dot{g}(\alpha)$ be the unique $\gamma$ so that there is $q$ 
in $\dot{G} \cap A_{\alpha}$ such that $\langle q, \gamma\rangle$ is in $X_{\alpha}$. Clearly $p$ forces that $\dot{g}=\dot{f}$, and $\dot{g}^{G}$ is in $M[G]$.

LEMmA 3.4. Suppose $M \subseteq N$ are transitive models of set theory with the same ordinals and $\lambda$ is a regular cardinal in $N$ such that $M$ is $\lambda$-closed in $N$. If $\mathbb{P}$ is a forcing poset in $M$ and $G$ is a generic filter for $\mathbb{P}$ over $M$ lying in $N$, then $M[G]$ is $\lambda$-closed in $N$.

Proof. In $N$ we have ${ }^{\lambda} O n \subseteq M \subseteq M[G]$.

Lemma 3.5. Suppose that $\kappa$ is a measurable cardinal, $\mathbb{P}$ is a forcing poset with size less than $\kappa$, and $U$ is a normal ultrafilter on $\kappa$. Then $\mathbb{P}$ forces that the filter $U^{*}$ generated by $U$ is a normal ultrafilter.

Proof. Let $j: V \rightarrow M=\operatorname{Ult}(V, U)$. Let $G$ be generic for $\mathbb{P}$ over $V$. Since $\operatorname{crit}(j)=\kappa, j(\mathbb{P})=j " \mathbb{P}$ and $j " G$ is generic for $j(\mathbb{P})$ over $M$. Extend $j$ to $j: V[G] \rightarrow M[j$ " $G]$. Define $U^{\prime}$ by letting $X \in U^{\prime}$ iff $X \subseteq \kappa$ and $\kappa \in j(X)$. Then $U^{\prime}$ is a normal ultrafilter containing $U$.

Let $U^{*}$ be the filter generated by $U$ in $V[G]$. Clearly $U^{*} \subseteq U^{\prime}$. To show that $U^{*}=U^{\prime}$ we show that $U^{*}$ is an ultrafilter. Suppose that $p$ is in $G$ and $p$ forces that $\dot{A} \subseteq \kappa$. Define a map $f: \kappa \rightarrow(\mathbb{P} / p \times 2)$ in $V$ by letting $f(\alpha)$ be some pair $\langle q, i\rangle$ such that $q \Vdash^{i}(\alpha \in \dot{A})$. Since $|\mathbb{P}|<\kappa$, there is $B$ in $U$ and $\langle q, i\rangle$ such that $f(\alpha)=\langle q, i\rangle$ for all $\alpha$ in $B$. But then $q$ forces that $B$ is a subset of $\dot{A}$ if $i=0$, and is a subset of the complement of $\dot{A}$ if $i=1$. So $U^{*}$ is an ultrafilter.

§4. Outline of the main theorem. We outline the argument for the construction of a model in which there is a strongly compact cardinal $\kappa$ such that $S\left(\kappa, \kappa^{+}\right)$is non-stationary.

Start with a model $V$ in which there is a supercompact cardinal $\kappa$ and GCH holds. Let $A$ be the set of $\alpha<\kappa$ such that $\alpha$ is measurable.

The basic idea of the proof is to add a club subset to $P_{\kappa} \kappa^{+}$which makes almost all $a$ in $S\left(\kappa, \kappa^{+}\right)$satisfy that $a \cap \kappa$ is in $A$, while at the same time destroying the stationarity of $A$.

We construct the model in two steps. In the first step we add a club to $P_{\kappa} \kappa^{+}$by forcing a partial square sequence. Since we will want to preserve the supercompactness of $\kappa$, we iterate such partial square posets for $\alpha$ in $A \cup\{\kappa\}$ using an Easton support iteration.

Let $W$ be a generic extension of $V$ by this iteration. Then in $W$, each $\alpha$ in $A$ is still a measurable cardinal. In the second step of the proof we use a Magidor iteration to add a Prikry sequence to each $\alpha$ in $A$. We show that the Magidor iteration destroys the stationarity of $A$ and preserves the strong compactness of $\kappa$. A key point is that by an absoluteness argument, the Magidor iteration will not change the properties of the partial square sequence added in the first stage.

$\S 5$. Iterating partial squares. In this section we complete the first step of the consistency proof as outlined above.

Start with a model $V$ in which GCH holds and there is a supercompact cardinal $\kappa$. Also assume that there is no inaccessible cardinal above $\kappa$. Let $A$ be the set of 
measurable cardinals less than $\kappa$. For each $\alpha$ in $A$ let $U_{\alpha}$ be a normal ultrafilter on $\alpha$ such that $A \cap \alpha$ is not in $U_{\alpha}$.

Define an Easton support forcing iteration $\mathbb{P}_{\kappa} * \mathbb{Q}_{\kappa}$ by induction as follows. Suppose that $\mathbb{P}_{\alpha}$ is defined for a fixed $\alpha \leq \kappa$. If $\alpha$ is not in $A \cup\{\kappa\}$ then let $\mathbb{Q}_{\alpha}$ be trivial. Suppose that $\alpha$ is in $A \cup\{\kappa\}$.

Let $G_{\alpha}$ be generic for $\mathbb{P}_{\alpha}$ over $V$. In $V\left[G_{\alpha}\right]$ let $B_{\alpha}$ be the set of limit ordinals $\beta<\alpha^{+}$such that $\operatorname{cf}(\beta)$ is less than $\alpha$ and $\operatorname{cf}(\beta)$ is not equal to $\mu^{+}$for any $\mu$ in $A \cap \alpha$.

Now let $\mathbb{Q}_{\alpha}$ be the forcing poset from Section 2 which adds a sequence $\left\langle c_{\beta}\right.$ : $\left.\beta \in B_{\alpha}\right\rangle$ such that:

(1) $c_{\beta}$ is club in $\beta$,

(2) $\operatorname{cf}\left(c_{\beta}\right)<\alpha$,

(3) if $\gamma \in \lim \left(c_{\beta}\right) \cap B_{\alpha}$, then $c_{\gamma}=c_{\beta} \cap \gamma$.

The poset $\mathbb{P}_{\alpha}$ forces that $\mathbb{Q}_{\alpha}$ is $(\alpha+1)$-strategically closed and has size $\alpha^{+}$.

This completes the definition of the iteration.

Suppose that $V_{1}$ is a generic extension of $V$ by $\mathbb{P}_{\kappa} * \mathbb{Q}_{\kappa}$. We show that for almost all $a$ in $S\left(\kappa, \kappa^{+}\right), a \cap \kappa$ is in $A$. Applying Lemma 2.2, let $C$ be a club subset of $P_{\kappa} \kappa^{+}$in $V_{1}$ such that for all $a$ in $C \cap S\left(\kappa, \kappa^{+}\right), a \cap \kappa$ is a cardinal and $\sup (a)$ has cofinality equal to $\mu^{+}$for some $\mu$ in $A$. Consider $a$ in $C \cap S\left(\kappa, \kappa^{+}\right)$. Then $\operatorname{cf}(\sup (a))=(a \cap \kappa)^{+}$. Since $a \cap \kappa$ is a cardinal, it follows that $a \cap \kappa$ is in A.

Proposition 5.1. For all $\alpha$ in $A, \mathbb{P}_{\kappa} * \mathbb{Q}_{\kappa}$ forces that there is a normal ultrafilter $U_{\alpha}^{*}$ on $\alpha$ such that $U_{\alpha} \subseteq U_{\alpha}^{*}$.

Proof. Write $\mathbb{P}_{\kappa} * \mathbb{Q}_{\kappa}=\mathbb{P}_{\alpha} * \mathbb{Q}_{\alpha} * \mathbb{P}_{\alpha, \kappa} * \mathbb{Q}_{\kappa}$. Let $G_{\alpha}$ be generic for $\mathbb{P}_{\alpha}$ over $V$. Since $\mathbb{Q}_{\alpha} * \mathbb{P}_{\alpha, \kappa} * \mathbb{Q}_{\kappa}$ does not add subsets to $\alpha$ over $V\left[G_{\alpha}\right]$, it suffices to prove that $U_{\alpha}$ can be lifted in $V\left[G_{\alpha}\right]$.

If $\alpha$ is not a limit point of $A$ then $\left|\mathbb{P}_{\alpha}\right|<\alpha$, and $U_{\alpha}$ can be lifted by Lemma 3.5. Suppose that $\alpha$ is a limit point of $A$.

In $V$ let $j_{\alpha}: V \rightarrow M_{\alpha}=\operatorname{Ult}\left(V, U_{\alpha}\right)$. Since $\alpha$ is not in $j_{\alpha}(A \cap \alpha), j_{\alpha}\left(\mathbb{P}_{\alpha}\right)$ factors as $\mathbb{P}_{\alpha} * \mathbb{P}_{\text {tail }}$ where $\mathbb{P}_{\text {tail }}$ is forced to be $\left(\min \left(j_{\alpha}(A \cap \alpha) \backslash(\alpha+1)\right)+1\right)$ strategically closed. The poset $\mathbb{P}_{\alpha}$ has size $\alpha$ and is $\alpha$-c.c., so has no more than $\alpha$-many antichains. Therefore $\mathbb{P}_{\text {tail }}$ has no more than $j_{\alpha}(\alpha)$-many antichains in $M_{\alpha}\left[G_{\alpha}\right]$. But $j_{\alpha}(\alpha)$ has size $\alpha^{+}$in $V\left[G_{\alpha}\right]$. So we can enumerate the set of maximal antichains of $\mathbb{P}_{\text {tail }}$ in $M_{\alpha}\left[G_{\alpha}\right]$ as $\left\langle A_{i}: i<\alpha^{+}\right\rangle$. Since $M_{\alpha}\left[G_{\alpha}\right]$ is $\alpha$ closed, $\mathbb{P}_{\text {tail }}$ is $\alpha^{+}$-strategically closed in $V\left[G_{\alpha}\right]$. So we can construct a generic $G_{\text {tail }}$ for $\mathbb{P}_{\text {tail }}$ over $M_{\alpha}\left[G_{\alpha}\right]$.

Now lift $j_{\alpha}$ to $j_{\alpha}: V\left[G_{\alpha}\right] \rightarrow M_{\alpha}\left[G_{\alpha} * G_{\text {tail }}\right]$. Define $U_{\alpha}^{*}$ by letting $X \in U_{\alpha}^{*}$ iff $X \subseteq \alpha$ and $\alpha \in j_{\alpha}(X)$. Then $U_{\alpha}^{*}$ is a normal ultrafilter on $\alpha$ with $U_{\alpha} \subseteq U_{\alpha}^{*}$.

Proposition 5.2. The poset $\mathbb{P}_{\kappa} * \mathbb{Q}_{\kappa}$ forces that $\kappa$ is supercompact. In fact, for all $\lambda>\kappa, \mathbb{P}_{\kappa} * \mathbb{Q}_{\kappa}$ forces that there is a normal ultrafilter on $P_{\kappa} \lambda$ which concentrates on $A$.

Proof. Fix $\lambda>\kappa^{+}$regular. Let $U$ be a normal ultrafilter on $P_{\kappa} \lambda$ and let $j: V \rightarrow M=\operatorname{Ult}(V, U)$. By the closure of $M, \kappa$ is measurable in $M$, so $\kappa$ is in $j(A)$. Write $j\left(\mathbb{P}_{\kappa}\right)=\mathbb{P}_{\kappa} * \mathbb{Q}_{\kappa} * \mathbb{P}_{\text {tail }}$. 
Let $G_{\kappa} * G$ be generic for $\mathbb{P}_{\kappa} * \mathbb{Q}_{\kappa}$. Since we assumed that there is no inaccessible above $\kappa, \min (j(A) \backslash(\kappa+1))>\lambda^{+}$. So $\mathbb{P}_{\text {tail }}$ is forced to be $\lambda^{+}$-strategically closed. Since $\mathbb{P}_{\kappa} * \mathbb{Q}_{\kappa}$ is $\kappa^{++}$-c.c., $M\left[G_{\kappa} * G\right]$ is $\lambda$-closed in $V\left[G_{\kappa} * G\right]$. Therefore $\mathbb{P}_{\text {tail }}$ is $\lambda^{+}$-strategically closed in $V\left[G_{\kappa} * G\right]$. In $M\left[G_{\kappa} * G\right], \mathbb{P}_{\text {tail }}$ has $j(\kappa)$ many maximal antichains. In $V\left[G_{\kappa} * G\right],|j(\kappa)|=\kappa^{\lambda}=\lambda^{+}$. So we can construct a generic filter $G_{\text {tail }}$ for $\mathbb{P}_{\text {tail }}$ over $M\left[G_{\kappa} * G\right]$. Extend $j$ to $j: V\left[G_{\kappa}\right] \rightarrow M\left[G_{\kappa} * G * G_{\text {tail }}\right]$.

We construct a master condition. For each $p$ in $G$ there is $\beta<\kappa^{+}$such that $p=\left\langle c_{\alpha}: \alpha \in B_{\kappa} \cap(\beta+1)\right\rangle$. So $j(p)=\left\langle d_{\alpha}: \alpha \in j\left(B_{\kappa}\right) \cap j(\beta+1)\right\rangle$. Let $s=\bigcup j$ " $G$. Then $s=\left\langle d_{\alpha}: \alpha \in j\left(B_{\kappa}\right) \cap \sup \left(j " \kappa^{+}\right)\right\rangle$. The sequence $s$ is a condition in $j\left(\mathbb{Q}_{\kappa}\right)$ iff the domain of $s$ is equal to $j\left(B_{\kappa}\right) \cap\left(\sup \left(j^{*} \kappa^{+}\right)+1\right)$, that is, iff $\sup \left(j^{\prime \prime} \kappa^{+}\right)$is not in $j\left(B_{\kappa}\right)$. But $\sup \left(j^{\prime \prime} \kappa^{+}\right)$has cofinality equal to the successor of $\kappa$, and $\kappa$ is a member of $j(A)$. So by the definition of $B_{\kappa}, \sup \left(j^{*} \kappa^{+}\right)$is not in $j\left(B_{\kappa}\right)$.

The poset $j\left(\mathbb{Q}_{\kappa}\right)$ has $j\left(\kappa^{++}\right)$many maximal antichains in $M\left[G_{\kappa} * G * G_{\text {tail }}\right]$. But $j\left(\kappa^{++}\right)$has size $\lambda^{+}$. In $M\left[G_{\kappa} * G * G_{\text {tail }}\right], j\left(\mathbb{Q}_{\kappa}\right)$ is $j(\kappa)$-strategically closed. But $M\left[G_{\kappa} * G * G_{\text {tail }}\right]$ is closed under $\lambda$-sequences and $|j(\kappa)|=\lambda^{+}$. So in $V\left[G_{\kappa} * G\right], j\left(\mathbb{Q}_{\kappa}\right)$ is $\lambda^{+}$-strategically closed. So we can construct a generic $H$ for $j\left(\mathbb{Q}_{\kappa}\right)$ which contains the master condition $s$. Now extend $j$ to $j: V\left[G_{\kappa} * G\right] \rightarrow$ $M\left[G_{\kappa} * G * G_{\text {tail }} * H\right]$.

Define $U^{*}$ by letting $X \in U^{*}$ iff $X \subseteq P_{\kappa} \lambda$ and $j^{\text {" }} \lambda \in j(X)$. Standard arguments show that $U^{*}$ is a normal ultrafilter extending $U$ in $V\left[G_{\kappa} * G\right]$.

§6. Magidor iteration. Let $W$ be a generic extension of $V$ by the poset $\mathbb{P}_{\kappa} * \mathbb{Q}_{\kappa}$ from the last section. Then in $W, \kappa$ remains supercompact and for every $\alpha$ in $A$ there is a normal ultrafilter $U_{\alpha}^{*}$ on $\alpha$ such that $A \cap \alpha$ is not in $A$.

We use a Magidor iteration to iterate Prikry forcing over $\alpha$ in $A$ using the ultrafilters $U_{\alpha}^{*}$. Define $\mathbb{M}_{\kappa}$ by recursion as follows. Our recursion hypothesis is that for each $\alpha$ in $A, \mathbb{M}_{\alpha}$ forces that there is a normal ultrafilter $U_{\alpha}^{* *}$ on $\alpha$ which extends $U_{\alpha}^{*}$.

Suppose that $\mathbb{M}_{\alpha}$ is defined for some $\alpha<\kappa$. If $\alpha$ is not in $A$ then force with the trivial poset at stage $\alpha$. If $\alpha$ is in $A$, then let $\mathbb{M}_{\alpha+1}=\mathbb{M}_{\alpha} * \mathbb{P} \mathbb{R}\left(U_{\alpha}^{* *}\right)$, where $\mathbb{P} R\left(U_{\alpha}^{* *}\right)$ is a name for the Prikry forcing defined from the ultrafilter $U_{\alpha}^{* *}$ which exists by the recursion hypothesis. Recall that Prikry forcing on $\alpha$ is a Prikry type forcing poset which satisfies the direct extension property and is $\alpha$-weakly closed.

Suppose that $\alpha$ is a limit ordinal and $\mathbb{M}_{\beta}$ is defined for all $\beta<\alpha$ using Prikry forcing at stages in $A \cap \alpha$. Define $\mathbb{M}_{\alpha}$ as follows. A condition in $\mathbb{M}_{\alpha}$ is a function $p$ with domain $\alpha$ such that for all $\beta<\alpha, p \uparrow \beta$ is in $\mathbb{M}_{\beta}$; moreover, there exists a finite set $a_{p} \subseteq \alpha$ such that $\beta$ is in $\alpha \backslash a_{p}$ iff $p\left\lceil\beta\right.$ forces that $p(\beta) \leq \leq^{*} 1$. We let $q \leq p$ if $q \uparrow \beta \leq p \uparrow \beta$ for all $\beta<\alpha$. Let $q \leq^{*} p$ if for all $\beta<\alpha$, $q \uparrow \beta \Vdash q(\beta) \leq^{*} p(\beta)$.

Such an iteration satisfies the Prikry property; see [8] or [3] for a proof.

Note that $\mathbb{M}_{\alpha}$ satisfies the direct extension property and is $\min (A)$-weakly closed.

LEMMA 6.1. If $\alpha$ is strongly inaccessible then $\mathbb{M}_{\alpha}$ is $\alpha^{+}$-c.c.

Proof. For each $\beta<\alpha,\left|\mathbb{M}_{\beta}\right|<\alpha$. By the direct extension property, if $p$ and $q$ are conditions such that $a_{p}=a_{q}$ and $p \uparrow a_{p}=q \uparrow a_{q}$, then $p$ and $q$ are 
compatible. There are $\alpha$ many possibilities for $a_{p}$, and given $a_{p}$, there are less than $\alpha$ many possibilities for $p\left\lceil a_{p}\right.$.

Suppose that $\alpha$ is in $A$. We verify the recursion hypothesis by showing that $\mathbb{M}_{\alpha}$ forces that $U_{\alpha}^{*}$ can be lifted.

If $\alpha$ is not a limit point of $A$ then $\left|\mathbb{M}_{\alpha}\right|<\alpha$ and all normal ultrafilters on $\alpha$ can be lifted by Lemma 3.5. Suppose that $\alpha$ is a limit point of $A$.

Proposition 6.2. Suppose that $G_{\alpha}$ is generic for $\mathbb{M}_{\alpha}$ over $W$. Then in $W\left[G_{\alpha}\right]$ there is a normal ultrafilter $U_{\alpha}^{* *}$ such that $U_{\alpha}^{*} \subseteq U_{\alpha}^{* *}$.

Proof. Let $j_{\alpha}: W \rightarrow M_{\alpha}=\operatorname{Ult}\left(W, U_{\alpha}^{*}\right)$. Since $\alpha$ is not in $j_{\alpha}(A)$, write $j_{\alpha}\left(\mathbb{M}_{\alpha}\right)=\mathbb{M}_{\alpha} * \mathbb{M}_{\text {tail }}$ where $\mathbb{M}_{\text {tail }}$ is forced to be $\alpha^{+}$-weakly closed.

In $W\left[G_{\alpha}\right]$ define $U_{\alpha}^{* *}$ by letting $X \in U_{\alpha}^{* *}$ iff $X \subseteq \alpha$ and there is an $\mathbb{M}_{\alpha}$-name $\dot{X}$ for $X$ and a condition $q \leq^{*} 1$ in $\mathbb{M}_{\text {tail }}$ such that $q$ forces over $M_{\alpha}\left[G_{\alpha}\right]$ that $\alpha \in j_{\alpha}(\dot{X})$.

First we show that the definition does not depend on the choice of $\dot{X}$. Suppose that $X=\dot{X}^{G_{\alpha}}=\dot{Y}^{G_{\alpha}}$. By the Prikry property there exists $q_{\dot{X}} \leq^{*} 1$ in $\mathbb{M}_{\text {tail }}$ which decides the statement $\alpha \in j_{\alpha}(\dot{X})$ and $q_{\dot{Y}} \leq^{*} 1$ which decides $\alpha \in j_{\alpha}(\dot{Y})$. We show that $q_{\dot{X}}$ and $q_{\dot{Y}}$ decide their respective statements the same way.

Suppose for a contradiction that $q_{\dot{X}} \Vdash \alpha \in j_{\alpha}(\dot{X})$ and $q_{\dot{Y}} \Vdash \alpha \notin j_{\alpha}(\dot{Y})$. Fix $p$ in $G_{\alpha}$ which forces that $\dot{X}=\dot{Y}$. Then $j_{\alpha}(p)$ forces that $j_{\alpha}(\dot{X}) \backslash j_{\alpha}(\dot{Y})$ is empty. Since $a_{p} \subseteq \alpha$ is finite, $j_{\alpha}\left(a_{p}\right)=a_{p}$. Therefore $j_{\alpha}(p)=p^{\widehat{c}} r$ where $r \leq^{*} 1$ in $\mathbb{M}_{\text {tail }}$. Note that $r$ forces over $M_{\alpha}\left[G_{\alpha}\right]$ that $j_{\alpha}(\dot{X}) \backslash j_{\alpha}(\dot{Y})$ is empty. By the direct extension property, there is $s$ which directly extends $r, q_{\dot{X}}$, and $q_{\dot{Y}}$. Then $s$ forces $\alpha \in j_{\alpha}(\dot{X}) \backslash j_{\alpha}(\dot{Y})$, contradicting the fact that $s \leq r$.

Similar arguments show that $U_{\alpha}^{* *}$ is a uniform ultrafilter extending $U_{\alpha}^{*}$. To show that it is normal, let $f: \alpha \rightarrow \alpha$ be a regressive function. Fix an $\mathbb{M}_{\alpha}$-name $\dot{f}$ for $f$ and $p$ in $G_{\alpha}$ which forces that $\dot{f}$ is regressive. Then $j_{\alpha}(p)$ forces that $j_{\alpha}(\dot{f}): j_{\alpha}(\alpha) \rightarrow j_{\alpha}(\alpha)$ is regressive. Write $j_{\alpha}(p)=p^{\widehat{ }} r$ where $r \leq^{*} 1$ in $\mathbb{M}_{\text {tail }}$. Then $r$ forces over $M_{\alpha}\left[G_{\alpha}\right]$ that $j_{\alpha}(\dot{f})$ is regressive. Using the $\alpha^{+}$-weak closure of $\mathbb{M}_{\text {tail }}$ in $M_{\alpha}\left[G_{\alpha}\right]$, define a $\leq^{*}$-decreasing sequence $\left\langle q_{i}: i \leq \alpha\right\rangle$, where $q_{0}=r$ and $q_{i+1}$ decides the statement $j_{\alpha}(\dot{f})(\alpha)=i$. Then $q_{\alpha}$ decides all such statements, so there must be some $\beta<\alpha$ such that $q_{\alpha} \Vdash j_{\alpha}(\dot{f})(\alpha)=\beta$. Let $\dot{X}$ be an $\mathbb{M}_{\alpha}$-name such that $p$ forces that $\dot{X}=\{\gamma<\alpha: \dot{f}(\gamma)=\beta\}$. Since $q_{\alpha} \leq r, q_{\alpha}$ forces that $\alpha \in j_{\alpha}(\dot{X})$. But $q_{\alpha} \leq^{*} 1$, so $\dot{X}^{G_{\alpha}}$ is in $U_{\alpha}^{* *}$.

This completes the definition of $\mathbb{M}_{\kappa}$.

Proposition 6.3. The poset $\mathbb{M}_{\kappa}$ forces that $\kappa$ is strongly compact.

Proof. Let $\lambda>\kappa$ be regular. In $W$ let $U$ be a normal ultrafilter on $P_{\kappa} \lambda$ which concentrates on $A$. Let $j: W \rightarrow M=\operatorname{Ult}(W, U)$. Since $\kappa$ is in $j(A)$, $j\left(\mathbb{M}_{\kappa}\right)=\mathbb{M}_{\kappa} * \mathbb{P R}\left(U_{\kappa}^{* *}\right) * \mathbb{M}_{\text {tail }}$. Note that $\mathbb{P R}\left(U_{\kappa}^{* *}\right) * \mathbb{M}_{\text {tail }}$ is forced to be $\kappa$ weakly closed.

Let $G_{\kappa}$ be generic for $\mathbb{M}_{\kappa}$. In $W\left[G_{\kappa}\right]$ define $U^{*}$ by letting $X \in U^{*}$ iff there is an $\mathbb{M}_{\kappa}$-name $\dot{X}$ for $X$ and $q \leq^{*} 1$ in $\mathbb{P R}\left(U_{\kappa}^{* *}\right) * \mathbb{M}_{\text {tail }}$ such that $q$ forces over $M\left[G_{\kappa}\right]$ that $j " \lambda \in j(\dot{X})$.

The proof that $U^{*}$ is a fine ultrafilter on $P_{\kappa} \lambda$ is similar to the proof of Proposition 6.2. We show that $U^{*}$ is $\kappa$-complete. Suppose that $p$ is in $G_{\kappa}$ and $p$ forces 
that $\left\langle\dot{X}_{i}: i<\beta\right\rangle$ is a partition of $P_{\kappa} \lambda$ for some $\beta<\kappa$. Then $j(p)$ forces that $j\left(\left\langle\dot{X}_{i}: i<\beta\right\rangle\right)=\left\langle j\left(\dot{X}_{i}\right): i<\beta\right\rangle$ is a partition of $j\left(P_{\kappa} \lambda\right)$. Since $j\left(a_{p}\right)=a_{p}$, $j(p)=p^{\widehat{r}} r$ where $r \leq^{*} 1$ in $\mathbb{P R}\left(U_{\kappa}^{* *}\right) * \mathbb{M}_{\text {tail }}$. Applying weak closure, find $q \leq^{*} r$ in $\mathbb{P R}\left(U_{\kappa}^{* *}\right) * \mathbb{M}_{\text {tail }}$ which decides the statements $j^{\text {" }} \lambda \in j\left(\dot{X}_{i}\right)$ for $i<\beta$. Since $r$ forces that $\left\langle j\left(\dot{X}_{i}\right): i<\beta\right\rangle$ is a partition of $j\left(P_{\kappa} \lambda\right)$ and $j$ " $\lambda$ is in $j\left(P_{\kappa} \lambda\right)$, there must be some $i<\beta$ such that $q$ forces that $j " \lambda \in j\left(\dot{X}_{i}\right)$. Then $\dot{X}_{i}^{G_{\kappa}}$ is in $U^{*}$. $\dashv$

Lemma 6.4. If $\mu$ is in $A$, then $\mathbb{M}_{\kappa}$ forces that both $\mu$ and $\mu^{+}$remain cardinals.

Proof. Write $\mathbb{M}_{\kappa}=\mathbb{M}_{\mu} * \mathbb{P} \mathbb{R}\left(U_{\mu}^{* *}\right) * \mathbb{M}_{\text {tail }}$. Then $\mathbb{M}_{\mu}$ preserves both $\mu$ and $\mu^{+}$ because it preserves the measurability of $\mu$ and is $\mu^{+}$-c.c. Prikry forcing preserves $\mu$ and $\mu^{+}$since it does not add bounded subsets to $\mu$ is $\mu^{+}$-c.c. Finally, $\mathbb{M}_{\text {tail }}$ is forced to be $\min (A \backslash(\mu+1))$-weakly closed so does not add subsets to $\mu^{+}$. $\dashv$

We now turn to the proof of the main theorem.

Let $G_{\mathbb{M}}$ be generic for $\mathbb{M}_{\kappa}$ over $W$. Then $\kappa$ is strongly compact in $W\left[G_{\mathbb{M}}\right]$. For each $\alpha$ in $A$, let $c_{\alpha}$ be the Prikry sequence added by $\mathbb{P} \mathbb{R}\left(U_{\alpha}^{* *}\right)$.

Proposition 6.5. Define $C_{\mathbb{M}}$ in $W\left[G_{\mathbb{M}}\right]$ as the set of $\beta<\kappa$ such that for all $\alpha \geq \beta$ in $A, \min \left(c_{\alpha}\right) \geq \beta$. Then $C_{\mathbb{M}}$ is a club subset of $\kappa$ disjoint from $A$.

Proof. If $\alpha$ is in $A$, then $\min \left(c_{\alpha}\right)<\alpha$, so $\alpha$ is not in $C_{\mathbb{M}}$. Suppose that $\delta<\kappa$ and $\left\langle\beta_{i}: i<\delta\right\rangle$ is an increasing sequence from $C_{\mathbb{M}}$. Let $\beta=\sup _{i<\delta} \beta_{i}$. If $\alpha \geq \beta$ is in $A$, then for each $i<\delta, \min \left(c_{\alpha}\right) \geq \beta_{i}$. So $\min \left(c_{\alpha}\right) \geq \sup _{i<\delta} \beta_{i}=\beta$.

To show that $C_{\mathbb{M}}$ is unbounded in $\kappa$, let $\beta<\kappa$ and let $p$ be some condition in $G_{\mathbb{M}}$. Fix $\xi$ in $\kappa \backslash A$ larger than $\max \left(a_{p}\right)$. Define $q$ by letting $q \uparrow(\xi+1)=p \uparrow(\xi+1)$, and for $\alpha$ in $A \backslash \xi$, let $q(\alpha)$ be a name for $p(\alpha) \backslash \xi$. Then $q$ forces that $\xi$ is in $C_{\mathbb{M}}$.

Suppose for a contradiction that $S\left(\kappa, \kappa^{+}\right)$is stationary in $W\left[G_{\mathbb{M}}\right]$. Let $\left\langle c_{\alpha}\right.$ : $\left.\alpha \in B_{\kappa}\right\rangle$ be the partial square sequence given by the generic for $\mathbb{Q}_{\kappa}$ in $W$. Recall that in $W, B_{\kappa}$ is the set of limit ordinals $\alpha<\kappa^{+}$with cofinality less than $\kappa$ such that the cofinality of $\alpha$ is not equal to $\mu^{+}$for any $\mu$ in $A$.

In $W\left[G_{\mathbb{M}}\right]$ fix $\theta \gg \kappa^{+}$regular. Since $S\left(\kappa, \kappa^{+}\right)$is stationary, there exists $N$ in $P_{\kappa} H(\theta)$ such that $N \cap \kappa^{+}$is in $S\left(\kappa, \kappa^{+}\right), N \cap \kappa$ is a limit cardinal, and $N$ is an elementary substructure of the model

$$
\left\langle H(\theta), \in, C_{\mathbb{M}},\left\langle c_{\alpha}: \alpha \in B_{\kappa}\right\rangle\right\rangle .
$$

Let $a=N \cap \kappa^{+}, \beta=\sup (a)$, and $\kappa_{a}=a \cap \kappa$. Note that $\kappa_{a}$ is in $C_{\mathbb{M}}$ and therefore $\kappa_{a}$ is not in $A$.

Claim 6.6. The ordinal $\beta$ is in $B_{\kappa}$.

Proof. If $\beta$ is not in $B_{\kappa}$, then in $W$ the cofinality of $\beta$ is $\mu^{+}$for some $\mu$ in $A$. In $W\left[G_{\mathbb{M}}\right], \mu^{+}$remains a cardinal and so the cofinality of $\beta$ is $\mu^{+}$. But the cofinality of $\beta$ in $W\left[G_{\mathbb{M}}\right]$ is equal to $\operatorname{cf}(a)=\kappa_{a}^{+}$. Since $\kappa_{a}$ and $\mu$ are cardinals, $\mu=\kappa_{a}$. This is a contradiction since $\mu$ is in $A$ but $\kappa_{a}$ is not in $A$.

Since $\beta$ is in $B_{\kappa}, c_{\beta}$ is defined and is a club subset of $\beta$ with order type less than $\kappa$. By elementarity, $B_{\kappa}$ is unbounded in $\beta$. Fix some uncountable regular cardinal $\delta<\kappa_{a}$ which is different from $\operatorname{cf}\left(\kappa_{a}\right)$ and is not the successor of a cardinal in $A$. Then $a$ is closed under suprema of subsets with order type $\delta$. 
Claim 6.7. The set $B_{\kappa}$ is closed under suprema of subsets with order type $\delta$.

Proof. Let $\gamma$ be the supremum of a subset of $B_{\kappa}$ with order type $\delta$. Then the cofinality of $\gamma$ in $W\left[G_{\mathbb{M}}\right]$ is $\delta$. If $\gamma$ is not in $B_{\kappa}$, then there is $\mu$ in $A$ such that $\mathrm{cf}^{W}(\gamma)=\mu^{+W}$. Since $\mu^{+W}$ is preserved, in $W\left[G_{\mathbb{M}}\right]$ we have $\operatorname{cf}(\gamma)=\mu^{+}$. So $\mu^{+}=\delta$, which contradicts the choice of $\delta$.

It follows that $a \cap B$ is closed under suprema of subsets with order type $\delta$, and in particular, $a \cap B$ is a stationary subset of $\alpha$. Since $c_{\beta}$ is unbounded in $\beta$, o.t. $\left(c_{\beta}\right) \geq \operatorname{cf}(\beta)=\kappa_{a}^{+}$. So there is $\gamma$ in $a \cap B \cap \lim \left(c_{\beta}\right)$ such that o.t. $\left(c_{\beta} \cap \gamma\right) \geq \kappa_{a}$. But $c_{\beta} \cap \gamma=c_{\gamma}$. Since $\gamma$ is in $N, c_{\gamma}$ is in $N$, and so also o.t. $\left(c_{\gamma}\right)$ is in $N \cap \kappa=\kappa_{a}$. This contradicts that o.t. $\left(c_{\gamma}\right) \geq \kappa_{a}$.

\$7. Strong Compactness and GCH. If $\kappa$ is supercompact and GCH holds for cardinals below $\kappa$, then GCH holds everywhere. Woodin asked whether the same is true for a strongly compact cardinal. In this section we prove a consistency result related to Woodin's problem. Using an argument similar to the proof of the main theorem above, we construct a model in which $\kappa$ is strongly compact, $2^{\left(\kappa^{+}\right)}=\kappa^{+++}$, but for a club of $\alpha$ below $\kappa, 2^{\left(\alpha^{+}\right)}=\alpha^{++}$.

Start with a model $V$ in which $\kappa$ is supercompact and GCH holds. Let $A$ be the set of measurable cardinals below $\kappa$.

Define an Easton support iteration $\mathbb{P}_{\kappa} * \mathbb{Q}_{\kappa}$ as follows. Suppose that $\mathbb{P}_{\alpha}$ is defined for some $\alpha \leq \kappa$. If $\alpha$ is not in $A \cup\{\kappa\}$ then let $\mathbb{Q}_{\alpha}$ be trivial. If $\alpha$ is in $A \cup\{\kappa\}$ then let $\mathbb{Q}_{\alpha}$ be a name for the poset $\operatorname{Add}\left(\alpha^{+}, \alpha^{+++}\right)$.

Arguments similar to those in Section 5 show that $\mathbb{P}_{\kappa} * \mathbb{Q}_{\kappa}$ preserves the measurability of each $\alpha$ in $A$ and normal ultrafilters on $P_{\kappa} \lambda$ which concentrate on $A$ can be lifted. Let $W$ be a generic extension of $V$ by $\mathbb{P}_{\kappa} * \mathbb{Q}_{\kappa}$. Then for all $\alpha$ in $A \cup\{\kappa\}, 2^{\left(\alpha^{+}\right)}=\alpha^{+++}$, and for any cardinal $\alpha$ not in $A \cup\{\kappa\}, 2^{\left(\alpha^{+}\right)}=\alpha^{++}$.

Let $\mathbb{M}_{\kappa}$ be the Magidor iteration of Prikry forcings defined over $\alpha$ in $A$. Let $G_{\mathbb{M}}$ be generic for $\mathbb{M}$ over $W$. Exactly as in Section $6, \kappa$ is strongly compact in $W\left[G_{\mathbb{M}}\right]$ and there is a club set $C_{\mathbb{M}}$ in $\kappa$ disjoint from $A$. Let $C$ be the club of limit cardinals in $C_{\mathbb{M}}$. Then $2^{\left(\kappa^{+}\right)}=\kappa^{+++}$, but for all $\alpha$ in $C, 2^{\left(\alpha^{+}\right)}=\alpha^{++}$.

REFERENCES

[1] J. Baumgartner, Iterated forcing, Surveys in set theory, Cambridge Univ. Press, 1983.

[2] J. Cummings, Iterated forcing and elementary embeddings, Preprint.

[3] M. GiTIK, Introduction to prikry type forcing notions, Preprint.

[4] - Nonsplitting subset of $P_{\kappa} \kappa^{+}$, J. Symbolic Logic, vol. 50 (1985), no. 4, pp. 881894.

[5] A. Kanamori, The higher infinite, Springer, 1994.

[6] J. Krueger, Adding clubs with square, Preprint.

[7] — Destroying stationary sets, Preprint.

[8] M. Magidor, How large is the first strongly compact cardinal?, Annals of Mathematical Logic, vol. 10 (1976), pp. 33-57. 
KURT GÖDEL RESEARCH CENTER FOR MATHEMATICAL LOGIC UNIVERSITY OF VIENNA

WÄHRINGERSTRASSE 25

1090 VIENNA, AUSTRIA

E-mail: jkrueger@logic.univie.ac.at

$U R L:$ http://www.logic.univie.ac.at/ jkrueger 